\title{
Quad-Active-Bridge as the basic cell of a MMC Based SST for DER and DESS Integration
}

\author{
Paul Frutos*, Fernando Briz ${ }^{\dagger}$, Alberto Sánchez*, and Juan Manuel Guerrero ${ }^{\dagger}$ \\ *Universidad San Francisco de Quito USFQ, Colegio de Ciencias e Ingeniería, Quito, Ecuador \\ Email: pfrutosg@usfq.edu.ec, asanchez@usfq.edu.ec \\ ${ }^{\dagger}$ Department of Electrical Engineering, Universidad de Oviedo, Spain \\ Email: fernando@isa.uniovi.es
}

\begin{abstract}
In a solid state transformer (SST), one of the most important roles is played by the dc-dc converter interfacing the high voltage port and the low voltage port while providing galvanic isolation. This paper proposes a quad-active-bridge (QAB) converter as the basic cell of a Modular Multilevel Converter (MMC) based solid state transformer for the integration of distributed energy resources (DER) and distributed energy storage systems (DESS) into the grid. This study comprises power transfer analysis, modeling and control of the QAB for this application. Furthermore, this paper shows an alternative method based on the star-mesh transformation to built the multiwinding tranformer equivalent circuit, that relates any two ports of the multiple-active-bridge (MAB) converter through a link inductance, which allows to describe power flow. Results are obtained by simulations in PLECS.
\end{abstract}

Keywords - Modular Multilevel Converter, MMC, solid state transformer, quad active bridge, multiple active bridge.

\section{INTRODUCTION}

The increasing penetration of renewable energies (e.g. photovoltaic and wind energy), the integration into the grid of new loads (e.g. electric vehicles), as well as the demanding requirements in terms of efficiency and reliability due to this integration in the power distribution system poses a big challenge, which is expected to increase in the near future [1], [2]. This scenario has pushed the development of innovative solutions based on high-power, high-voltage electronics power converters, like Flexible AC Transmission Systems (FACTS), High Voltage Direct Current (HVDC), and Solid State Transformers, which should be able to cope with the new challenges [3]. The SST is envisioned as an important piece for future smartgrid applications that require an efficient integration of distributed generation and storage resources, flexible routing mechanisms and active filtering [4].

The SST's, a power electronics-based technology, consist of one or more medium frequency (MF) transformers that perform power conversion with galvanic isolation. They are considered an alternative to the conventional low-frequency transformers because they are able to provide functionalities to the power operator such as power flow control, reduction of transmission losses, power quality improvement, harmonics and imbalance compensation, availability of low-voltage DC (LVDC) link, and smart protection. Many different topologies for Solid State Transformer have been proposed and developed by academia and industry like the Modular Multilevel
Converter based SST and the Cascade H-Bridge based SST. [4], [5].

The MMC based SST is composed by a modified modular multilevel converter capable of transferring power (inject/drain) within the cells. Fig. 1 shows the constitutive cells of each arm of the MMC converter. Each cell typically consist of a half bridge and a capacitor, and medium frequency DC/DC converters connected in parallel to the MMC cell capacitors. A multiple active bridge converter (MAB) such as dual active bridge (DAB) or a $\mathrm{QAB}$ can be used for this purpose [2], [6].

Existing control methods for MMC converters with power transfer capable cells assume that all the cells have the same design and operate in the same conditions. This implies that both DC and AC components of the overall voltages are evenly split among cells. Since the current is common to all the cells in each arm, imbalances in the transferred power by the cells will necessarily result in imbalances among the cell voltages [4], [6]. Therefore, when DABs are used to inject/drag power to/from the MMC cells, they must transfer the same amount of power in order to avoid these imbalances. That is fine when all the DABs are parallelized to form a low voltage high current DC link, but this is not the case when DER or DESS are connected (e.g. photovoltaic panels receive a different amount of solar radiation, batteries operate with different states of charge).

This paper proposes a MMC based STT using QAB converters as the basic cells of the DC/DC conversion stage as shown in Fig. 1, where each QAB converters is connected to two MMC cells while the third port of each QAB could be used to connect either a DER or DESS, the fourth port of each DC/DC converter must be connected to the LVDC bus that could interacts with a low voltage AC (LVAC) grid by means of a power inverter (e.g. four-leg voltage source converter [7]). This figure exemplifies a SST with four MMC cells per leg (i.e. two MMC cells per arm) which are connected to QAB converters. The first $\mathrm{QAB}$ is located in the upper arm of the A-phase leg, with a port connected to a PV energy source. The second QAB located in the lower arm of the same leg/phase is connected to a battery system.

The paper is organized in the following way: section II shows the derivation of the mesh equivalent circuit of a MAB converter. Section III presents the power flow analysis of 


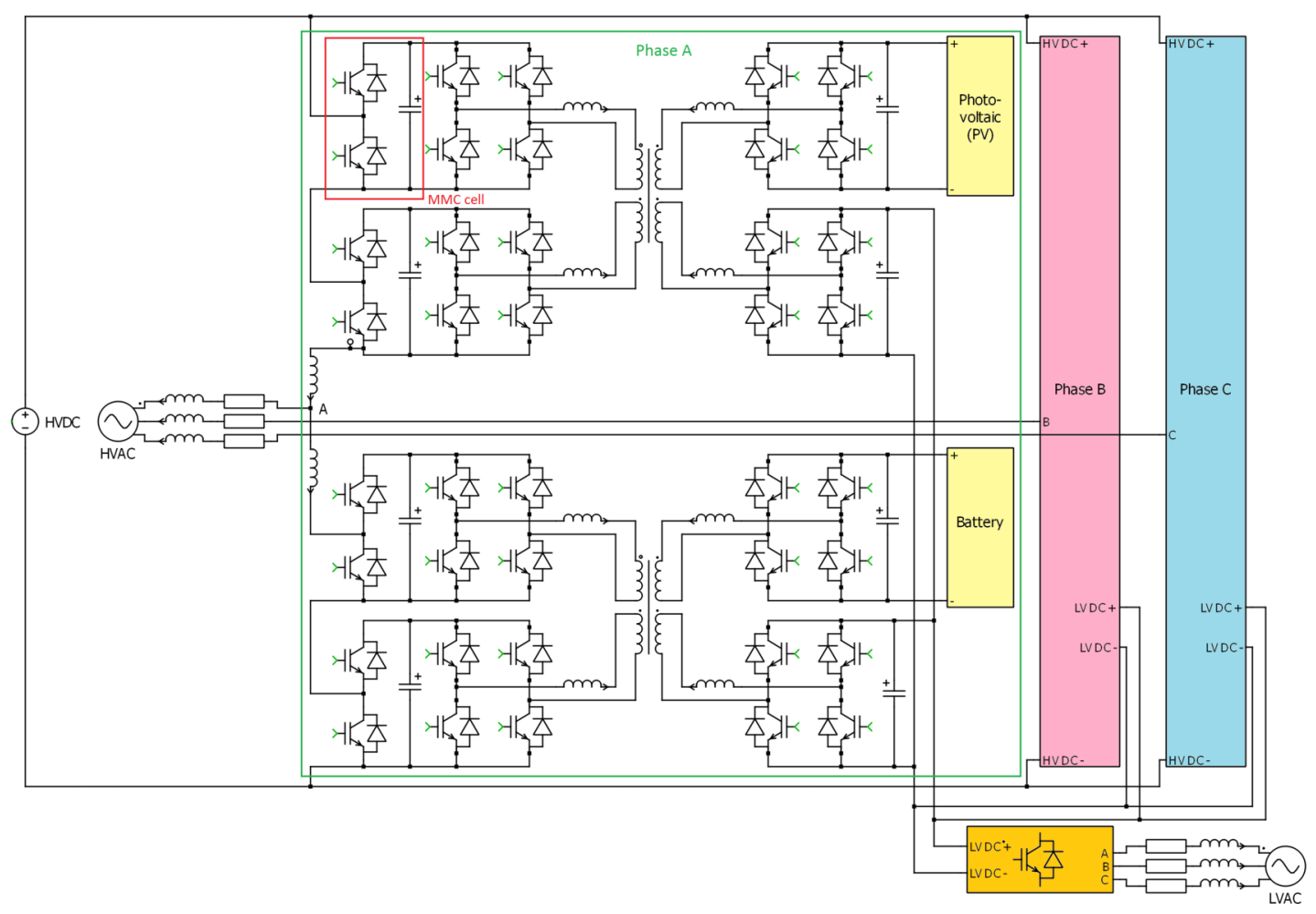

Fig. 1: MMC based SST using the QAB converter as the basic cell of dc/dc stage for DER and/or DESS integration

the $\mathrm{QAB}$ transferring power from/to the MMC cells when a DER/DESS is connected. Section IV presents the control theory for the QAB in this application. Section V presents and discusses simulation results of the MMC based SST focusing on the QAB converters behavior and the MMC cells associated. Finally in section VI, conclusions are drawn.

\section{Multiple Active Bridge: Mesh Equivalent CIRCUIT}

To perform a power flow analysis of a MAB it is necessary to find an equivalent circuit that relates any two ports of the MAB. This section presents an alternative method based on the star-mesh transformation to find an equivalent circuit for the multiwinding transformer.

\section{A. Mesh Equivalent Circuit Derivation}

A MAB consists of $n$-full-bridge converters magnetically coupled through a $n$-winding medium frequency transformer. The QAB is a particular case of the MAB when $n=4$ as shown in Fig. 2, where the self-leakage inductances of the windings are $L_{k}$, the voltages at the transformer terminals are $v_{k}$, and currents through the transformer windings are $i_{k}$, and $k$ varies from 1 to $n$. To simplify the analysis it is common to neglect the mutual leakage inductances in medium frequency transformers as in [8]. Then, the circuit equations at the $k$ th winding of the transformer can be written as in equation (1), where $e_{k}$ is the electromotive force in the $k$-th winding produced by the main magnetic flux linkage.

$$
v_{k}-e_{k}=L_{k} \frac{d i_{k}}{d t}
$$

In order to ease the circuit analysis of any MAB converter, the self-leakage inductance $L_{k}$, the voltages and currents of each winding/port can be referred to winding 1 according to the set of equations in (2), where $N_{k}$ is the number of turns of the the winding $k$.

$$
v_{k}^{\prime}=\left(\frac{N_{1}}{N_{k}}\right) v_{k}, i_{k}^{\prime}=\left(\frac{N_{k}}{N_{1}}\right) i_{k}, L_{k}^{\prime}=\left(\frac{N_{1}}{N_{k}}\right)^{2} L_{k}
$$

Then, equation (1) can be written as:

$$
v_{k}^{\prime}-e=L_{k}^{\prime}\left(d i_{k}^{\prime} / d t\right)
$$

From this equation it is possible to define the "star" equivalent circuit of any multiple winding transformer, where $e=L_{m}\left(d i_{m} / d t\right)$ and $i_{m}=\sum_{k=1}^{n} i_{k}$. The star equivalent circuit of a QAB is shown in Fig. 3.

The mesh equivalent circuit of a $n$-winding/ports MAB can be built using the $n+1$ degree star-mesh transformation [9], where the ground counts as an extra node. This is an alternative method to the one presented in [8]. The mesh equivalent circuit relates any two ports of a MAB through a link inductance $L_{j k}$ which allows to perform a power transfer analysis. This inductance can be calculated using equation (4) and the shunt inductance $L_{g k}$ of each MAB port is given by equation (5). The mesh equivalent circuit of a QAB is shown in Fig. 4. It is important to notice that the calculation of link inductance $L_{j k}$ using equation (4) is straightforward and easy to understand, 
whereas in [8] this derivation is more complex and does not consider the shunt inductances.

$$
\begin{aligned}
& L_{j k}=L_{j}^{\prime} L_{k}^{\prime}\left(\sum_{i=1}^{n} \frac{1}{L_{i}^{\prime}}+\frac{1}{L_{m}}\right) \\
& L_{g k}=L_{k}^{\prime} L_{m}\left(\sum_{i=1}^{n} \frac{1}{L_{i}^{\prime}}+\frac{1}{L_{m}}\right)
\end{aligned}
$$

For this particular study, it is considered that each QAB winding has the same number of turns, so $N_{1}=N_{2}=$ $N_{3}=N_{4}$ and $L_{1}=L_{2}=L_{3}=L_{4}=L$. Therefore, the link inductances and the shunt inductances are defined as $L_{j k}=L_{Q}=L\left(L+4 L_{m}\right) / L_{m}$ and $L_{g k}=4 L_{m}+L$. Considering $L_{m}>L$, then $L_{Q}=4 L$ and the shunt inductances $L_{g k}$ can be neglected in the circuit model.

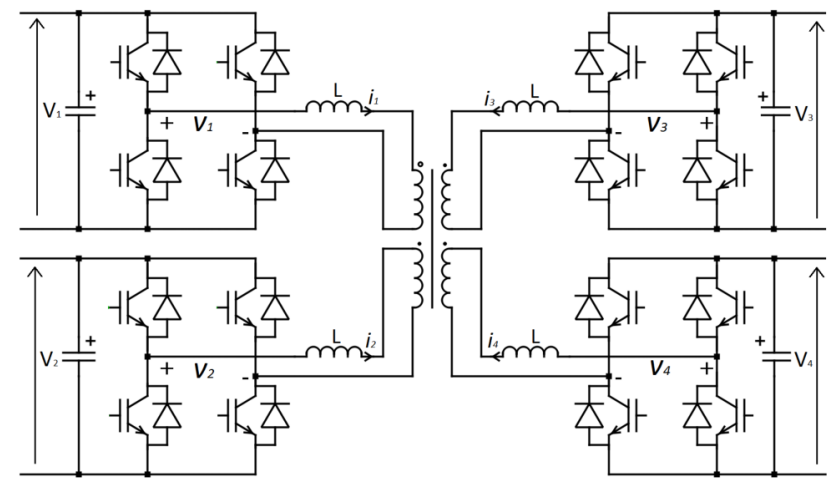

Fig. 2: Quad active bridge converter schematic

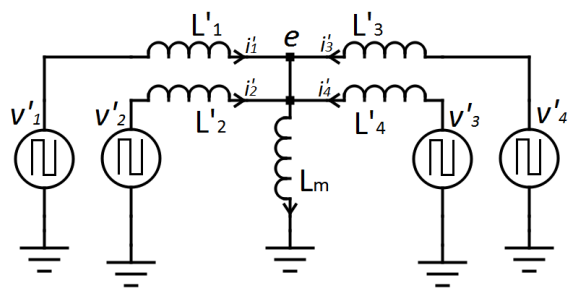

Fig. 3: QAB "star" equivalent circuit

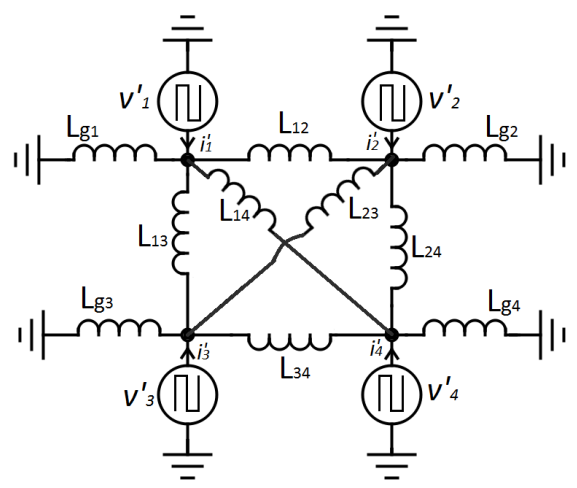

Fig. 4: QAB "mesh" equivalent circuit for power transfer analysis

\section{POWER Transfer ANALYSis}

This section presents the power flow analysis of the QAB when it is operating as the cross-link of the MMC based SST. This analysis is performed in two steps. First, the power flow equations are derived for a single $\mathrm{QAB}$, and later these are extended to the $\mathrm{QAB}$ integrated within the MMC converter. An example is provided at the end of the section.

\section{A. Quad Active Bridge: Power Transfer Analysis}

The power flow between any two ports of the QAB when controlled with simple-phase-shift (SPS) modulation [10] is given by the following equation.

$$
P_{i j}=\frac{V_{i} V_{j}}{2 \pi f_{s}\left(L_{Q}\right)} \phi_{i j}\left(1-\frac{\left|\phi_{i j}\right|}{\pi}\right), \phi_{i j}=\phi_{i}-\phi_{j}
$$

where $\phi_{k}$ is the phase shift angle of the voltage $v_{k}$. The power transfer between any two ports of the QAB can be regulated using the phase shift angle difference $\phi_{i j}$. Equation (7) states the power conservation principle. Therefore, there are three degrees of freedom to perform the power flow control action.

$$
0=P_{1}+P_{2}+P_{3}+P_{4}
$$

where $P_{j}=\sum_{k \neq j} P_{j k}$. The max power transfer of each link is:

$$
P_{\text {link_max }}=\frac{V_{i} V_{j}}{4 f_{s}\left(L_{Q}\right)}
$$

The QAB converter can operate in some scenarios according to the specific application (e.g 3-sources-1-load or 2-sources2-loads), but always under the constraints of equations (6) and (7)

\section{B. Quad Active Bridge - MMC based SST: Power Transfer Analysis}

In this study, the QAB converter is always transferring an equal amount of power through the two ports connected to the MMC cells while the others ports are free for power transfer whilst maintaining the power constraint equations (6) and (7). So, the voltage phase-shift angles from port 1 and port 2 must be the same and they are set to zero for reference (e.g. $\phi_{1}=$ $\phi_{2}=0$ ), which also implies that these ports do not exchange power between them. As a consequence of this, the threedegree of freedom of a usual QAB converter is reduced to two and the power flow of each port can be controlled by the voltage phase-shift angles $\phi_{3}$ and $\phi_{4}$. Fig. 5 shows the amount of power that each port is injecting or draining to/from the MF transformer as a function of $\phi_{3}$ and $\phi_{4}$, where $D_{3}=$ $\phi_{3} / \pi$ and $D_{4}=\phi_{4} / \pi$ are the phase shift ratios corresponding to the port 3 and port 4 respectively. In these figures, it is important to notice that the values of power are normalized to per unit values. The normalized power is defined as $P_{\text {norm }}=$ $2 P_{\text {link_max }}$.

Since the power transferred to the converter by ports 1 and 2 is the same, Fig. 5a applies to both ports. The maximum power that these ports handle is 1 [p.u], that is the case when $D_{3}=D_{4}= \pm 0.5$. On the other hand, port 3 and port 4 
can inject or drain a maximum power of 1.5 [p.u], again, all the cases are under the energy balance constraint given by equations (6) and (7).

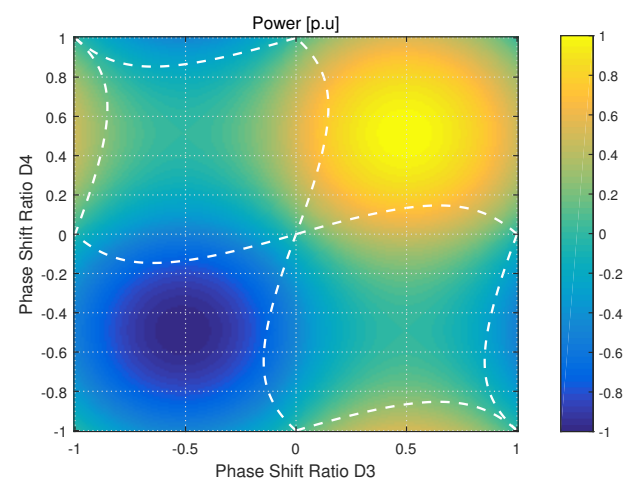

(a)

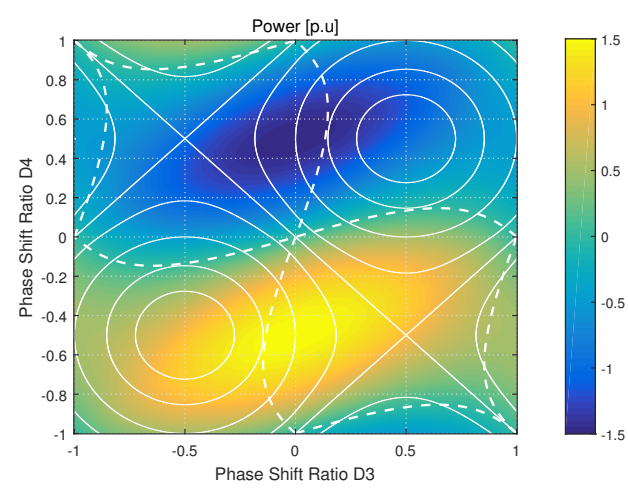

(b)

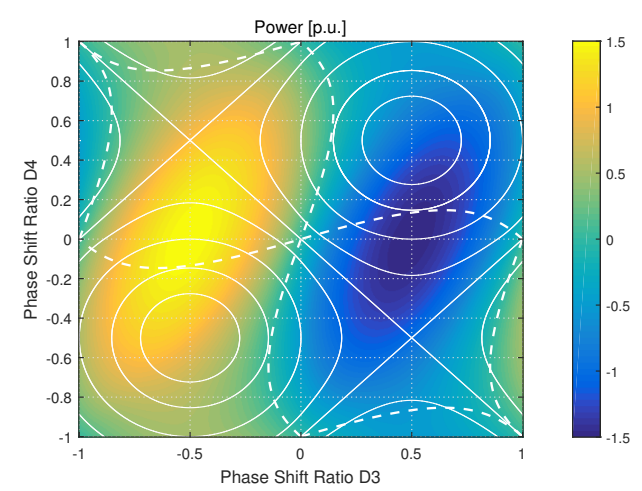

(c)

Fig. 5: Power transfer characteristic map (a) port 1/port 2, (b) port 3 (c) port 4. White dashed line is the limit of the operational regions. Continuous white lines are the level curves of port 1

Fig. 6 shows the operational regions of the QAB as a function of $D_{3}$ and $D_{4}$. In the yellow region, 2 ports send power and 2 ports receive power. The red dashed line is the 2-sources-1-load/1-source-2-loads scenario and the blue region is the 3-sources-1-load/1-source-3-loads scenario.Also, It is important to notice the contour map (i.e. level curves) of Fig. $5 \mathrm{a}$ is presented in Fig. 6. This contour map is replicated in the power transfer characteristic map of Port 3 and Port 4 in

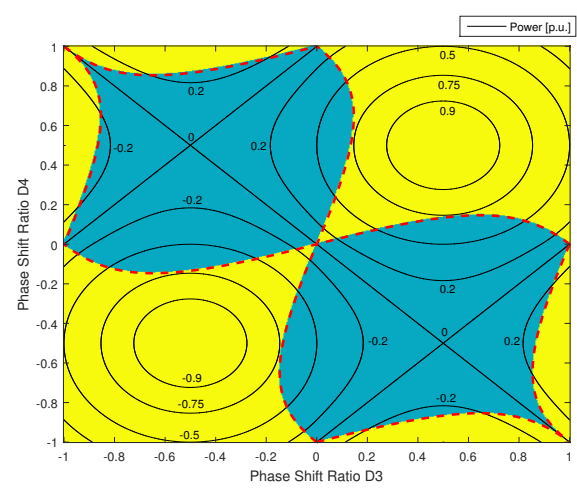

Fig. 6: QAB Operational Regions. Yellow region (2-sources-2-loads). Red dashed line (2-sources-1-load/1-source-2-loads). Blue region (3-sources-1-load/1-source-3-loads).

order to easy power flow analysis, see Fig. $5 \mathrm{~b}$ and $5 \mathrm{c}$.

\section{Example Case:}

Consider ports 1 and 2 deliver 0.75 [p.u], so the two ports are feeding the QAB converter with 1.5 [p.u]. This operation point is located on the 0.75 [p.u] level curve shown in Fig. $5 b$ and $5 \mathrm{c}$. Now, the total injected power into the system has to be drained by port 3 and port 4 , leading to several possible scenarios, three of them are presented. A first scenario would be when port 3 drains 1.5 [p.u.] and port 4 drains 0 [p.u.], that happens when $D_{3}=0.14$ and $D_{4}=0.5$. On the contrary case, when port 4 evacuates all the power $D_{3}=0.5$ and $D_{4}=0.14$. A third scenario would be when port 3 and port 4 evacuate the same amount of power, in that case $D_{3}=D_{4}=0.25$.

\section{CONTROL StRategies}

The control strategies for the different stages of the MMC based SST are described in this section. First, a brief explanation of the MMC converter control is given, then a control technique for a $\mathrm{QAB}$ converter connected to the cells of the MMC converter and used for distributed energy and storage integration is widely presented. These control techniques will be used later in the simulation study of section $\mathrm{V}$.

\section{A. MMC converter Control}

The control of the MMC converter is a challenging task since multiple control requirements need to be satisfied simultaneously. Among these are the generation of the output AC voltage, the control of the circulating current and the balancing of the cells capacitors [11]. Modulation and control strategies developed for modular multilevel converters are mainly aimed to balance the power between the AC and DC ports performed by balancing of the cell capacitor voltages and by controlling the circulating current in the MMC legs [12].

\section{B. Quad Active Bridge Control}

1) Current Control: From power transfer equation (6) and assuming that the voltage values in each port of the QAB are established, the dc current component $I_{j k}$ is obtained using 
equation (9). So, the total dc current component of port $j$ is given by equation (10).

$$
\begin{gathered}
I_{j k}=\frac{P_{j k}}{V_{j}}=\frac{V_{k}}{2 \pi f_{s} L_{Q}} \phi_{j k}\left(1-\frac{\left|\phi_{j k}\right|}{\pi}\right) \\
I_{j}=\sum_{k \neq j}^{4} I_{j k}=I_{j}\left(\phi_{1}, \phi_{2}, \phi_{3}, \phi_{4}\right)
\end{gathered}
$$

Equation (10) shows the QAB as a non-linear multiple input multiple output (MIMO) system. Equations (11)-(13) describe the linearized model (i.e small signal model) of the plant around the operational point $O P$ [8], where $\mathbf{H}$ is a $4 \times 4$ linearized matrix, $\boldsymbol{\delta} \mathbf{I}=\left[\begin{array}{llll}\delta I_{1} & \delta I_{2} & \delta I_{3} & \delta I_{4}\end{array}\right]^{T}$ is the small variation on the current vector, and $\delta \Phi=$ $\left[\begin{array}{llll}\delta \phi_{1} & \delta \phi_{2} & \delta \phi_{3} & \delta \phi_{4}\end{array}\right]^{T}$ is a small variation on the phase shift angle vector.

$$
\begin{gathered}
\boldsymbol{\delta} \mathbf{I}=\mathbf{H}\left(\phi_{1_{O P}}, \phi_{2_{O P}}, \phi_{3_{O P}}, \phi_{4_{O P}}\right) \boldsymbol{\delta} \boldsymbol{\Phi} \\
H_{j j}=\left.\frac{\partial I_{j}}{\partial \phi_{j}}\right|_{O P}=\sum_{l \neq j}^{4} \frac{V_{l}}{2 \pi f_{s} L_{Q}}\left[1-\frac{2\left|\phi_{j_{O P}}-\phi_{l_{O P}}\right|}{\pi}\right] \\
H_{j k}=\left.\frac{\partial I_{j}}{\partial \phi_{k}}\right|_{O P}=-\frac{V_{k}}{2 \pi f_{s} L_{Q}}\left[1-\frac{2\left|\phi_{j_{O P}}-\phi_{k_{O P}}\right|}{\pi}\right]
\end{gathered}
$$

Since the $\mathrm{QAB}$ is a three degree of freedom system, the reduced linear system model shown in equation (14) is enough to represent the plant model, and it is useful to design the control of the dynamic response of the system, where $\mathbf{H}_{\mathbf{R}}$ is the $3 \times 3$ reduced matrix of $\mathbf{H}$

$$
\left[\begin{array}{l}
\delta I_{1} \\
\delta I_{2} \\
\delta I_{3}
\end{array}\right]=\mathbf{H}_{\mathbf{R}}\left(\phi_{1_{O P}}, \phi_{2_{O P}}, \phi_{3_{O P}}, \phi_{4_{O P}}\right)\left[\begin{array}{c}
\delta \phi_{2} \\
\delta \phi_{3} \\
\delta \phi_{4}
\end{array}\right]
$$

Due to all the inputs and all the outputs of the system are coupled, a decoupling operation at the plant input can be implemented using the inverse matrix of $\mathbf{H}_{\mathbf{R}}$ [13], which is denoted as $\mathbf{J}$, see equation (15). Equation (16) shows the interaction of the control loops can be eliminated, where I_e, defined as the estimated value of the current vector, is the new input of the system. So, we can treat the system as three independent control loops, proportional-integral (PI) controllers are used. The schematic of the closed control loop with the decoupling stage is shown in Fig. 7.

$$
\begin{aligned}
& {\left[\begin{array}{lll}
\delta \phi_{2} & \delta \phi_{3} & \delta \phi_{4}
\end{array}\right]^{T}=\mathbf{J}\left[\begin{array}{lll}
\delta I_{1 \_} e & \delta I_{2 \_} e & \delta I_{3 \_} e
\end{array}\right]} \\
& {\left[\begin{array}{l}
\delta I_{1} \\
\delta I_{2} \\
\delta I_{3}
\end{array}\right]=\mathbf{H}_{\mathbf{R}} \times \mathbf{J} \times\left[\begin{array}{l}
\delta I_{1 \_} e \\
\delta I_{2 \_} e \\
\delta I_{3 \_} e
\end{array}\right]}
\end{aligned}
$$

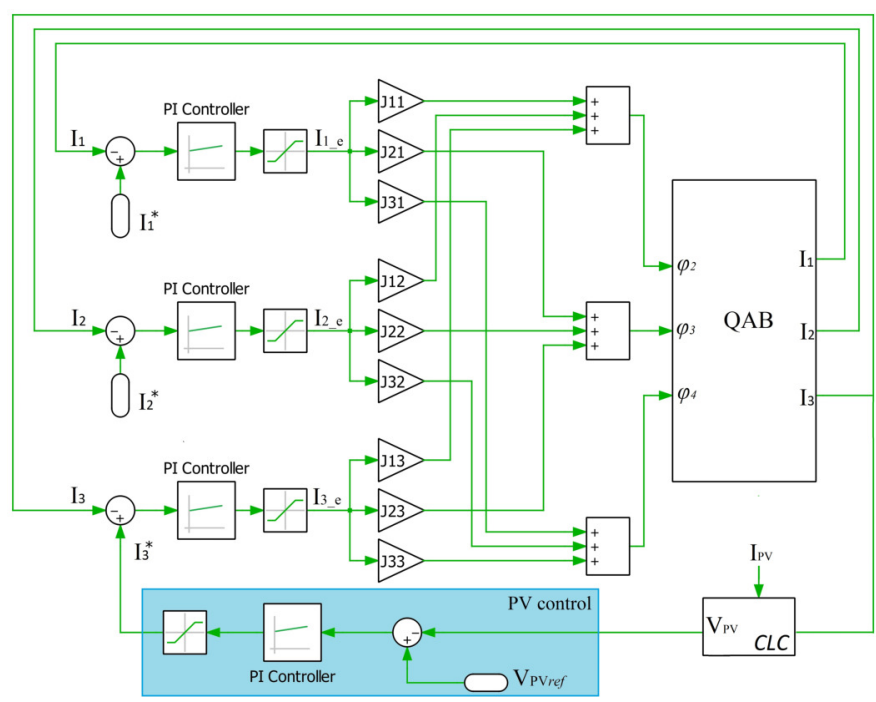

Fig. 7: QAB Closed Control Loop - Integration of PV energy source

2) Power Control: Assuming that the voltage in the ports of the QAB are established the control of the power can be reached simply by computing the amount of the current reference value necessary to get the reference power $I_{j}^{*}=V_{j} / P_{j}^{*}$. Since the capacitor voltage of the MMC is a controlled value, then the reference current set at port 1 and 2 of each QAB is

$$
I_{1,2}^{*}=\frac{V_{M M C_{-} \text {cell }}}{P_{\text {cell }}^{*}}
$$

3) PV Voltage control: A PV source can be considered and modeled as a current source, so for this port, it is required to perform a voltage control action in order to set the PV voltage. A CLC filter can be employed to support the voltage control action and to prevent damage of the PV panels due to the ripple currents from the converter. So, the PV voltage can be regulated to a reference value provided for instance by a maximum power point tracking system [14]. It is important to notice that the inner port current controller has to be much faster than the PV voltage controller. A anti-wind up system can also be included. Equation (18) is the CLC filter transfer function which gives its dynamic behavior. Fig. 7 shows the closed control loop schematic for this control action.

$$
\begin{gathered}
\frac{V_{P V}(s)}{I_{3}(s)}=\frac{1}{L C_{1} C_{2} s^{3}+\left(C_{1}+C_{2}\right) s^{2}} \\
I_{3}^{*}=\frac{k_{p} s+k_{i}}{s}\left(V_{P V \_r e f}^{*}-V_{P V}\right)
\end{gathered}
$$

4) Battery current control: On the other hand, batteries do not require a voltage control since they tend to maintain a constant voltage value in all its operational region. Although it is possible to decide if the battery is charging or discharging by controlling the port current, which is already done, the ripple currents can cause severe problems, LC filters are added to the ports and a controller is designed in order to avoid abrupt 
changes in the current. The battery current reference value could be calculated from a management algorithm [15]. For this case, a lead-lag compensator is used.

$$
\begin{gathered}
\frac{I_{b a t t}(s)}{I_{3}(s)}=\frac{1}{1+L_{b a t t} C_{b a t t} s^{2}} \\
I_{3}^{*}=k \frac{\left(s+\alpha_{1}\right)\left(s+\alpha_{2}\right)}{\left(s+\beta_{1}\right)\left(s+\beta_{2}\right)}\left(I_{b a t t}^{*}-I_{b a t t}\right)
\end{gathered}
$$

\section{Simulation Study}

This section presents a simulation study of the $\mathrm{QAB}$ acting as the dc/dc stage of the MMC based SST performed in the software PLECS. The switch model of the SST as shown in Fig. 1, but with a larger number of MMC cells, was built and tested in this software. The parameters of the system are presented in Table I

TABLE I: MMC based SST Parameters

\begin{tabular}{|l|l|l|l|}
\hline \multicolumn{2}{|l|}{ MMC Parameters } & \multicolumn{2}{l|}{ QAB Parameters } \\
\hline VacHV & $24 \mathrm{kV}$ & Max. Power & $15 \mathrm{~kW}$ \\
\hline VdcHV & $16 \mathrm{kV}$ & Max. Power per port & $5 \mathrm{~kW}$ \\
\hline$P$ cell & $5 \mathrm{~kW}$ & Switching freq. & $20 \mathrm{kHz}$ \\
\hline P. Total & $240 \mathrm{~kW}$ & Turns ratio & $1: 1: 1: 1$ \\
\hline MMC cells leg & 16 & Leakage inductance & $160 \mathrm{uH}$ \\
\hline QABs total & 24 & VdcLV Link & 1000 \\
\hline
\end{tabular}

1) Photo-voltaic energy source integration: A PV is connected to the $\mathrm{QAB}$, which transfers power from the MMC cells to the LVDC bus. Therefore, ports 1 and 2 of the QAB are injecting the same amount of power, whilst port 3 feeds power from the PV source and port 4 drains all this power to the LVDC bus.

The control action and the dynamic response of the QAB is shown in Fig. 8. Initially, the MMC cells are injecting 5KW through ports 1 and 2, the same amount of power is being transferred by the PV source, so port 4 has to drain $15 \mathrm{KW}$. The QAB remains in this initial state until a step change of $-2.5 \mathrm{KW}$ in the power fed by the PV source occurs at time 0.3 seconds. It is observed in this figure that the control system is capable of regulating the power in all the ports after the disturbance. The power difference is managed by port 4, connected to the LV grid while the controller keeps constant the power in the ports connected to the MMC cell capacitor. Also, the PV voltage controller is capable of maintaining the required voltage. The controller is tested again at time 0.4 seconds.

Fig. 9 shows the capacitor voltage time response of the MMC cells that are connected to the QAB. From this figure, it can be seen that although ports 3 and 4 are transferring unbalanced power with oscillations. The voltage at the MMC cells capacitors remain balanced with very small oscillations.
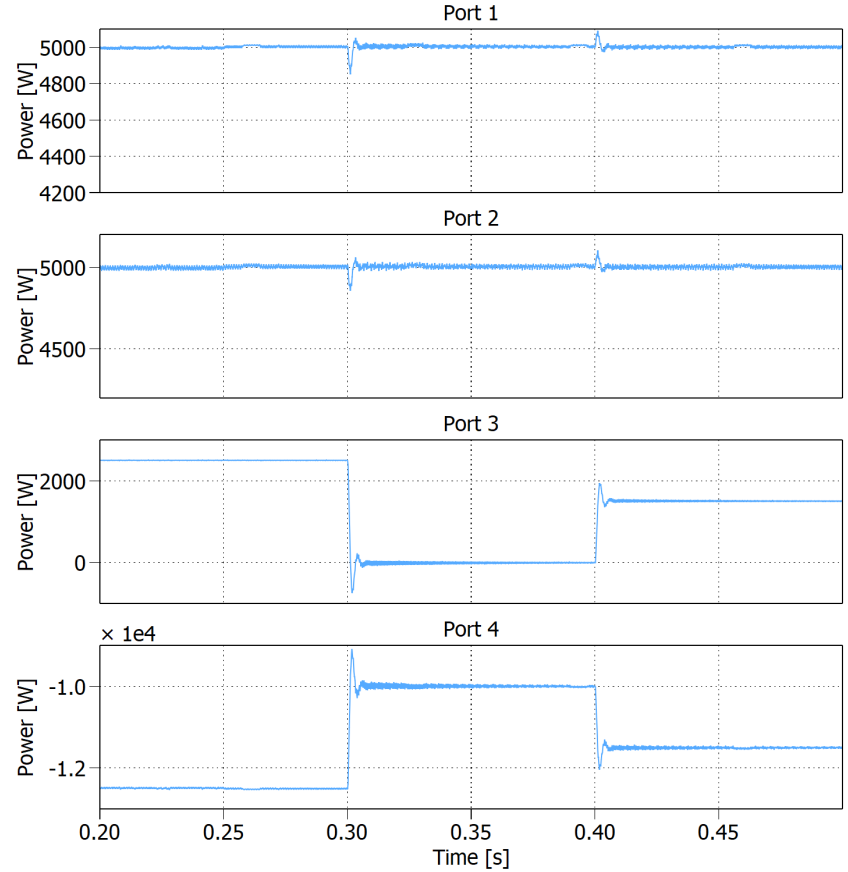

(a)

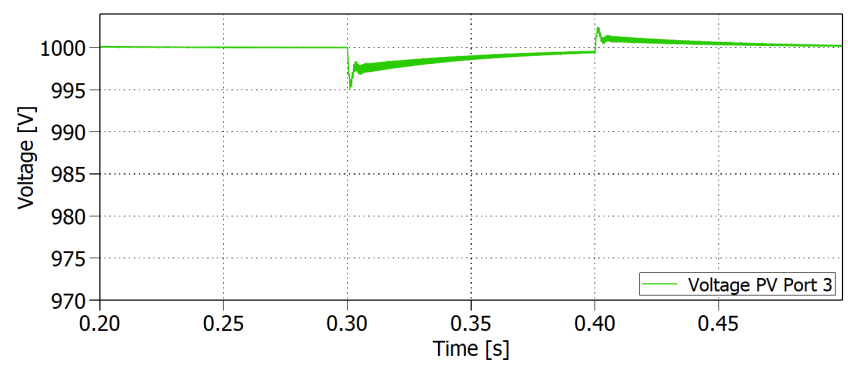

(b)

Fig. 8: System Time Response: (a) Power Control,(b) PV Voltage Control

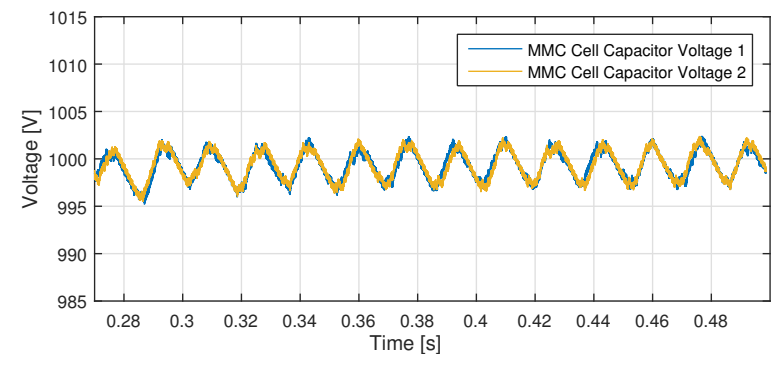

Fig. 9: Capacitor Voltages in two cells of the MMC

2) Battery system integration: Fig. 11 shows the the battery current time response for two step changes at times $0.3 \mathrm{~s}$ and $0.4 \mathrm{~s}$. It is possible to see that the current controller is able to perform the control task when references in the current are changed from $5 \mathrm{~A}$ to $2.5 \mathrm{~A}$ and from $2.5 \mathrm{~A}$ to $5 \mathrm{~A}$. Fig. 12 shows the steady state battery current and the steady state port current, here it is possible to notice the LC circuit response, which avoids the large current ripple. The current control 


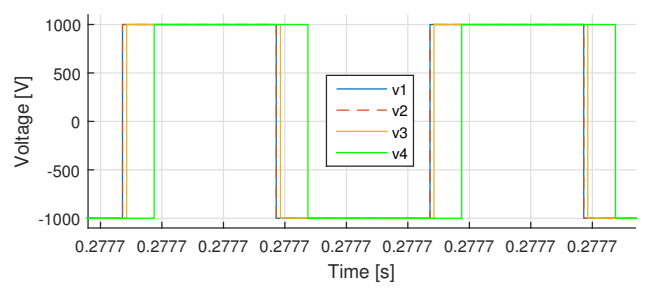

(a)

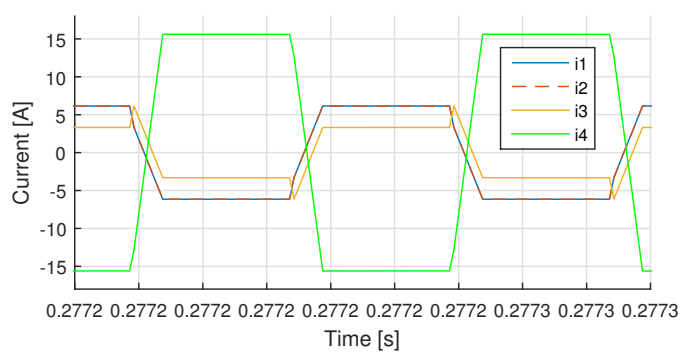

(b)

Fig. 10: Steady State Waveforms, (a) Voltage (b) Current

regulates the battery current almost at a constant value in the steady state.

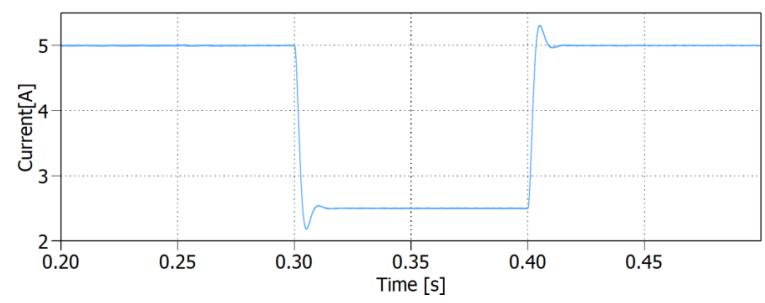

Fig. 11: Battery Current Time Response
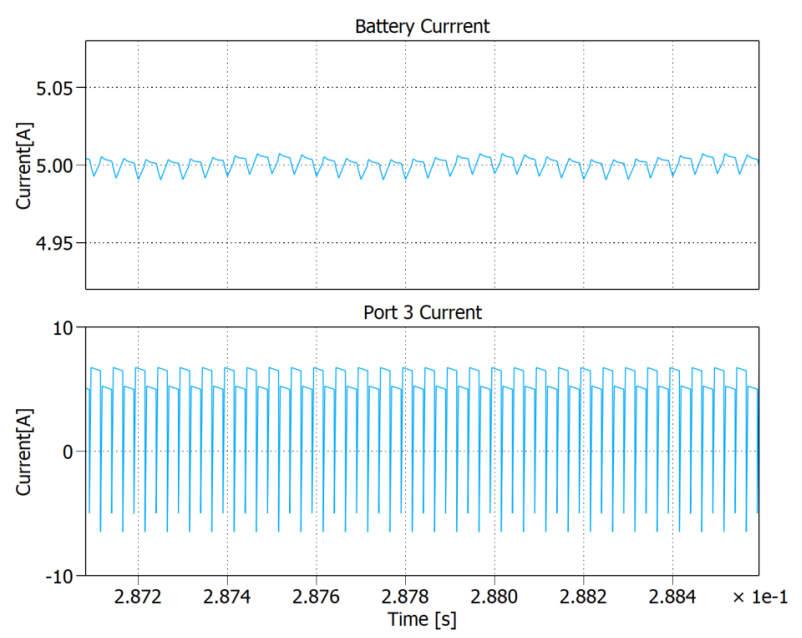

Fig. 12: Steady state current waveform, Battery current and port current

\section{CONCLUSIONS}

This paper proposes a $\mathrm{QAB}$ as the cross-link cell of the DC/DC stage of the MMC based SST. The QAB is able to integrate DER and DESS while maintaining the same power transfer in the MMC cells, so the voltages in the capacitors of the MMC cells remain balanced. The power transfer analysis shows the different operational regions of the QAB converter and how the power can be transferred among its ports. The required controllers are designed using the system small signal model. The performance of the proposed solid state transformer topology is verified by simulation. Finally, it is important to highlight that the star-mesh transformation is used to build the MAB equivalent circuit in order to perform a power flow analysis.

\section{REFERENCES}

[1] B. Gemmell, J. Dorn, D. Retzmann, and D. Soerangr, "Prospects of multilevel vsc technologies for power transmission," in 2008 IEEE/PES Transmission and Distribution Conference and Exposition, April 2008, pp. 1-16.

[2] F. Briz, M. Lopez, A. Zapico, A. Rodrguez, and D. Daz-Reigosa, "Operation and control of mmcs using cells with power transfer capability," in 2015 IEEE Applied Power Electronics Conference and Exposition (APEC), March 2015, pp. 980-987.

[3] M. Lopez, A. Rodrguez, E. Blanco, M. Saeed, . Martnez, and F. Briz, "Design and implementation of the control of an mmc-based solid state transformer," in 2015 IEEE 13th International Conference on Industrial Informatics (INDIN), July 2015, pp. 1583-1590.

[4] F. Briz, M. Lopez, A. Rodriguez, and M. Arias, "Modular power electronic transformers: Modular multilevel converter versus cascaded hbridge solutions," IEEE Industrial Electronics Magazine, vol. 10, no. 4, pp. 6-19, Dec 2016.

[5] J. E. Huber and J. W. Kolar, "Solid-state transformers: On the origins and evolution of key concepts," IEEE Industrial Electronics Magazine, vol. 10, no. 3, pp. 19-28, Sep. 2016.

[6] F. Briz, M. Lopez, A. Rodriguez, A. Zapico, M. Arias, and D. DiazReigosa, "Mmc based sst," in 2015 IEEE 13th International Conference on Industrial Informatics (INDIN), July 2015, pp. 1591-1598.

[7] P. Frutos, E. Christopher, A. Sanchez, and O. Aguirre, "A performance comparison of stationary frame control of three-leg and four-leg voltage source inverters in power system applications," in IECON 2018 - 44th Annual Conference of the IEEE Industrial Electronics Society, Oct 2018, pp. 925-931.

[8] S. Falcones, R. Ayyanar, and X. Mao, "A dcdc multiport-converter-based solid-state transformer integrating distributed generation and storage," IEEE Transactions on Power Electronics, vol. 28, no. 5, pp. 2192-2203, May 2013.

[9] M. van Lier and R. Otten, "Planarization by transformation," IEEE Transactions on Circuit Theory, vol. 20, no. 2, pp. 169-171, March 1973.

[10] B. Zhao, Q. Song, W. Liu, and Y. Sun, "Overview of dual-active-bridge isolated bidirectional dcdc converter for high-frequency-link powerconversion system," IEEE Transactions on Power Electronics, vol. 29, no. 8, pp. 4091-4106, Aug 2014.

[11] M. Lopez, F. Briz, A. Zapico, A. Rodriguez, and D. Diaz-Reigosa, "Control strategies for mmc using cells with power transfer capability," in 2015 IEEE Energy Conversion Congress and Exposition (ECCE), Sep. 2015, pp. 3570-3577.

[12] M. Hagiwara and H. Akagi, "Control and experiment of pulsewidthmodulated modular multilevel converters," IEEE Transactions on Power Electronics, vol. 24, no. 7, pp. 1737-1746, July 2009.

[13] C. Zhao and J. W. Kolar, "A novel three-phase three-port ups employing a single high-frequency isolation transformer," in 2004 IEEE 35th Annual Power Electronics Specialists Conference (IEEE Cat. No.04CH37551), vol. 6, June 2004, pp. 4135-4141 Vol.6.

[14] S. Shi, Y. Wang, and P. Jin, "Study of maximum power point tracking methods for photovoltaic power generation system," in 2013 Chinese Automation Congress, Nov 2013, pp. 835-840.

[15] B. V. Lukutin, E. B. Shandarova, and I. L. Fuks, "Energy efficiency of photovoltaic power plants in stand-alone power supply systems," in 2016 2nd International Conference on Industrial Engineering, Applications and Manufacturing (ICIEAM), May 2016, pp. 1-5. 